Article

\title{
Hepcidin-Expressing Fish Eggs as A Novel Food Supplement to Modulate Immunity against Pathogenic Infection in Zebrafish (Danio rerio)
}

\author{
Chung-Chih Tseng ${ }^{1,2,+}$, Tah-Wei Chu ${ }^{3,+}$, Ridha Handriany Danata ${ }^{4,5}$, Yenny Risjani ${ }^{5}$, \\ Hui-Tsu Shih ${ }^{6, *}$ and Shao-Yang $\mathrm{Hu}^{4,7, *(D)}$ \\ 1 Department of Dentistry, Zuoying Branch of Kaohsiung Armed Forces General Hospital, \\ Kaohsiung 81357, Taiwan; caviton@g-mail.nsysu.edu.tw \\ 2 Institute of Medical Science and Technology, National Sun Yat-Sen University, Kaohsiung 80424, Taiwan \\ 3 Department and Graduate Institute of Aquaculture, National Kaohsiung University of Science and \\ Technology, Kaohsiung 811, Taiwan; twchu@nkust.edu.tw \\ 4 Department of Biological Science and Technology, National Pingtung University of Science and Technology, \\ Pingtung 91201, Taiwan; rd.danata@gmail.com \\ 5 Department of Fisheries and Marine Science, University of Brawijaya, Malang 65145, Indonesia; \\ risjani@ub.ac.id \\ 6 Department of Clinical Pharmacy, Zuoying Branch of Kaohsiung Armed Forces General Hospital, \\ Kaohsiung 81357, Taiwan \\ 7 Research Center for Animal Biologics, National Pingtung University of Science and Technology, \\ Pingtung 91201, Taiwan \\ * Correspondence: shz530812@gmail.com (H.-T.S.); syhu@mail.npust.edu.tw (S.-Y.H.) \\ + Chung-Chih Tseng and Tah-Wei Chu contributed equally to this work.
}

Received: 27 March 2020; Accepted: 14 May 2020; Published: 15 May 2020

Abstract: Hepcidin antimicrobial peptides are difficult to produce in prokaryotic expression systems due to their complex structure and antimicrobial activity. Although synthetic hepcidin provides an alternative to solve this issue, its high cost limits its practical application in various industries. The present study used zebrafish eggs as bioreactors to produce convict cichlid (Amatitlania nigrofasciata) hepcidin (AN-hepc) using the oocyte-specific zona pellucida (zp3) promoter. The expression plasmid pT2-ZP3-AN-hepc-ZP3-EGFP, using EGFP as a reporter of AN-hepc expression, was designed to establish the transgenic line Tg(ZP3:AN-hepc:ZP3:EGFP) for the expression of AN-hepc. The AN-hepc peptide was produced successfully in fertilized eggs, as evidenced by RT-PCR and Western blotting. The AN-hepc-expressing eggs exhibited antimicrobial activity against a variety of aquatic pathogens and antibiotic-resistant pathogens, suggesting that the AN-hepc expressed in fish eggs was bioactive. The immunomodulatory effects of AN-hepc-expressing fertilized eggs on zebrafish innate immunity were evaluated by determining the expression of indicator genes after feeding with AN-hepc-expressing fertilized eggs for two months. Zebrafish supplementation with AN-hepc-expressing fertilized eggs significantly increased the expression of innate immunity-related genes, including IL-1 $\beta$, IL-6, IL-15, TNF- $\alpha$, NF-kb, complement C3b, lysozyme and TLR-4a. The zebrafish administered AN-hepc-expressing eggs exhibited higher cumulative survival than fish supplemented with wild-type and control eggs after infection with Aeromonas hydrophila and Streptococcus iniae. In conclusion, the present results showed that supplementation with AN-hepc-expressing fish eggs enhanced zebrafish innate immunity against pathogenic infections, suggesting that fertilized eggs containing AN-hepc have the potential to be developed as a food supplement for improving health status in aquaculture.

Keywords: hepcidin; bioreactor; innate immunity; disease resistance; zebrafish 


\section{Introduction}

Fish diseases caused by bacterial pathogens result in high mortality of cultured fish and substantial economic loss, making them one of the major causes impeding the sustainable development of aquaculture. For example, Aeromonas hydrophila and Streptococcus iniae are typical pathogens that cause red fin disease and hemorrhagic septicemia in several freshwater fish species [1,2]. To address these diseases, antibiotic and antimicrobial chemicals are widely used in aquaculture for prophylactic and therapeutic purposes. However, the abuse of antibiotic and antimicrobial chemicals has led to the rapid spread of antimicrobial-resistant pathogens, which impacts on microbial ecology and food-related risks for human health due to residual chemicals in aquatic products. In light of this issue, alternatives to antibiotics for disease control are urgently needed. Antimicrobial peptides (AMPs), as critical components of innate immunity, exert antimicrobial activity and immunomodulatory functions to enhance piscine host defenses against pathogenic infection. Thus, AMPs offer a promising alternative for the modulation of immunity in aquatic animals for disease control [3].

Hepcidin is a small cysteine-rich cationic AMP that was first isolated from human urine and named based on its primary expression in hepatocytes and antimicrobial properties [4]. In addition to having antimicrobial activity, hepcidin also plays an important role in a variety of physiological functions, including controlling iron homeostasis [5], depleting extracellular iron to protect against infection by invading pathogens [6], inhibiting iron metabolism by causing degradation, and occluding transportation of ferroportin (Fpn), the only known cellular iron exporter in vertebrates [7]. Since the first piscine hepcidin was isolated from hybrid striped bass [8], hepcidin genes have been isolated and characterized in more than 40 species of teleost fish [3,9-14]. Among these studies, several reports have shown that the hepcidin genes are expressed in the liver and immune tissues and respond to pathogen challenge and inflammation, suggesting that their physiological function is primarily associated with immunity [15-18]. This hypothesis was verified by examining the antimicrobial activities and immunomodulatory effects of hepcidin in various fish using synthetic hepcidin peptides. However, the use of synthetic hepcidin may not completely reveal the structure and biological activity of native hepcidin. Furthermore, the high cost of synthetic hepcidin is unsuitable for practical aquaculture applications. Investigators have attempted to produce hepcidin using a prokaryotic expression system such as recombinant E. coli. However, even though large amounts of AMPs are produced in bacterial cells, almost all recombinant hepcidin is expressed as insoluble inclusion bodies due to the presence of complex disulfide bonds [19]. Although bioactive hepcidin peptide can be obtained from inclusion bodies by denaturation, refolding and purification procedures, the complexity of the process usually increases the cost and time consumption. Investigations have attempted to solve this issue by fusing hepcidin with highly soluble partner proteins [20,21]. Pan reported that epinecidin fused with DsRed protein was expressed as soluble form, and the soluble fusion protein-expressed E. coli could be used as immunostimulant by oral administration [22]. However, in certain cases, the partner proteins may cause inactivation of the target hepcidin [23]. Moreover, the separation of hepcidin from its fusion partner for purifying hepcidin using proteolytic enzymes is highly expensive. Thus, although hepcidin peptides are efficient immunostimulators of fish against bacterial infection, in practice, it is difficult to obtain sufficient hepcidin peptide for disease control in aquaculture. Given this issue, a practical and low-cost strategy to acquire enough bioactive hepcidin is necessary.

The use of transgenic fish as bioreactors is an emerging application for the production of recombinant proteins. Herein, zebrafish provided an excellent model due to a series of advantages, including the ease and relatively low cost of maintenance, short generation time (approximately 3 months), spawning capability throughout the year under optimal temperature control (approximately $28^{\circ} \mathrm{C}$ ), ability to acquire large numbers of fertilized eggs (200 300 eggs) from each pair, ability to achieve complex post-translational modification of eukaryotic proteins, and no virus that can infect humans and the fish. In addition, gene transfer using the Tol2 transposon system can be easily manipulated in zebrafish, which exhibits high germline transmission efficiency. For example, human coagulation factor VII (hVFII) was successfully expressed in zebrafish embryos 
by the cytomegalovirus (CMV) promoter, suggesting that human therapeutic proteins can potentially be produced in fish eggs [24]; bovine lactoferricin (LFB), ubiquitously expressed in zebrafish under the $\beta$-actin promoter, demonstrates that bactericidal peptides can effectively be produced in zebrafish eggs for use as a supplement to improve fish immunity against pathogenic infection [25]; tilapia insulin-like growth factor (IGF)-1 and -2 could be biologically produced and purified from zebrafish eggs, suggesting that the use of fish eggs as bioreactors is a promising strategy for the production of heterologous proteins [26]. These examples highlight the fact that transgenic zebrafish, as bioreactors, can produce highly valuable recombinant proteins in a practical and economical manner.

In our previous study, the convict cichlid (Amatitlania nigrofasciata) hepcidin gene (AN-hepc) was cloned from liver tissue. The expression of AN-hepc was demonstrated to be significantly induced in the liver and most tissues after lipopolysaccharide (LPS) challenge. The synthetic AN-hepc peptide showed a wide spectrum of antimicrobial activity in vitro against a variety of pathogens and revealed in vivo efficacy in the improvement of disease resistance in pathogen-infected zebrafish [18]. To practically and economically apply AN-hepc in aquaculture, the present study aimed to use zebrafish eggs as bioreactors for the production of bioactive AN-hepc peptide using the oocyte-specific zona pellucida (zp3) promoter. The antimicrobial activity against pathogens and immunomodulatory function of AN-hepc-expressing zebrafish eggs in fish were evaluated in zebrafish. We expected that the results could provide reference values for practical applications of AN-hepc in aquaculture.

\section{Materials and Methods}

\subsection{Fish and Bacterial Strains}

Adult AB strain wild-type (WT) zebrafish (Danio rerio) were obtained from the Taiwan Zebrafish Core Facility at Academia Sinica (Taipei, Taiwan). The fish were reared with acclimation in a 120-L freshwater recirculating aquarium at $28^{\circ} \mathrm{C}$ with a controlled light cycle of $14 \mathrm{~h} \mathrm{light} / 10 \mathrm{~h}$ dark and fed twice daily with a commercial diet (MeM Prime, BERNAQUA, Olen, Belgium). All fish were treated according to local animal welfare regulations. The experimental protocol was approved (approval no. NPUST-108-019) by the Institutional Animal Care and Use Committee (IACUC) of NPUST. Zebrafish embryos used for microinjection were acquired from intercross mating of the $\mathrm{AB}$ strain zebrafish. The source and origin of indicator pathogens, culture conditions and maintenance of pathogens were performed as described in previous reports [27-29].

\subsection{Construction of the AN-hepc Expression Plasmid}

The pT2-ZP3-NTR-EGFP vector, including the expression cassette (zona pellucida (zp3) promoter, nitroreductase ( $n t r)$ gene, egfp gene, SV40 polyadenylation signal) and flanks to the expression cassette of Tol2 transposon arms (500 bp left arm and $500 \mathrm{bp}$ right arm), was used as the backbone to construct the expression plasmid [30]. A sequence fragment, which comprised the mature AN-hepc gene, 6 histidine tag and SV40 polyadenylation signal, was synthesized and cloned into pT2-ZP3-NTR-EGFP to replace the $n t r$ gene between the AgeI and EcoRI restriction sites, thereby generating the construct pT2-ZP3-AN-hepc-EGFP. A 2.2-kb 5' flanking region of the zona pellucida gene, which is capable of driving oocyte-specific expression, was synthesized and cloned into pT2-ZP3-AN-hepc-EGFP between the EcoRI and BamHI restriction sites to generate the expression plasmid pT2-ZP3-AN-hepc-ZP3-EGFP, in which AN-hepc and EGFP expression were triggered by two independent $z p 3$ promoters. Moreover, a synthetic EGFP gene was cloned into the pT2-ZP3-NTR-EGFP vector between the AgeI and ClaI restriction sites to generate the pT2-ZP3-EGFP control plasmid, in which EGFP was expressed alone under the $z p 3$ promoter.

\subsection{Establishment of Transgenic Zebrafish Lines}

Transgenic zebrafish lines were generated by the Tol2 transposon system, which was isolated from medaka (Oryzias latipes) and encoded a functional transposase capable of catalyzing transposition 
and improving the transgenic efficiency of germline transmission. The pCS-TP plasmid encoding the Tol2 transposase was kindly provided by Dr. Kawakami [31]. Linearization of pCS-TP was performed by NotI, and the linearized DNA was used as a template for preparing Tol2 transposase mRNA by using the mMessage mMachine Kit (Ambion, Foster City, USA). To generate stable Tg(ZP3:EGFP) and Tg(ZP3:AN-hepc:ZP3:EGFP) lines, $100 \mathrm{ng} / \mu \mathrm{l}$ pT2-ZP3-EGFP and pT2-ZP3-AN-hepc-ZP3-EGFP plasmids, respectively, were coinjected with $100 \mathrm{ng} / \mu \mathrm{l}$ capped Tol2 transposase mRNA into one-cell-stage embryos. The injected embryos were reared to adults, which, with oocyte-specific expression of EGFP, were considered transgenic female founders. F1 transgenic offspring were obtained from the intercrossed transgenic founders by detecting EGFP expression using fluorescence microscopy. The F2 transgenic eggs from intercrossed F1 transgenic fish were used to characterize AN-hepc expression and feeding trials.

\subsection{Detection of AN-hepc in Fertilized Eggs}

For detecting protein expression of AN-hepc in fertilized eggs, two hundred fertilized eggs at $8 \mathrm{~h}$ postfertilization (hpf) were washed with $500 \mu \mathrm{L}$ of PBS buffer $(140 \mathrm{mM} \mathrm{NaCl}, 2.7 \mathrm{mM} \mathrm{KCl}, 10 \mathrm{mM}$ $\mathrm{Na}_{2} \mathrm{HPO}_{4} \_12 \mathrm{H}_{2} \mathrm{O}, 1.8 \mathrm{mM} \mathrm{KH}_{2} \mathrm{PO}_{4} ; \mathrm{pH}$ 7.3). The washed fertilized eggs were suspended in $200 \mu \mathrm{L}$ PBS buffer and homogenized with a small plastic pestle in a $1.5 \mathrm{~mL}$ microcentrifuge tube. The supernatant was collected by centrifugation at $10,000 \times \mathrm{g}$ for $10 \mathrm{~min}$ at $4{ }^{\circ} \mathrm{C}$, and then determined the protein concentration by the Bradford protein assay method using BSA as the standard. A 10 ug of supernatant protein was separated by $16.5 \%$ Tricine SDS-PAGE, and then the blot was electrophoretically transferred to a PVDF membrane using Bio-Rad protein mini gel apparatus. The membranes were blocked overnight at $4{ }^{\circ} \mathrm{C}$ in Tris-buffered saline containing $0.1 \%$ Tween 20 and $5 \%$ skimmed milk. The blots were probed with anti-mouse 6x-his tag monoclonal antibody (MA1-21315, Thermo), anti-rabbit GFP polyclonal antibody (GTX 113617, GeneTex) and anti-rabbit $\beta$-actin polyclonal antibody (GTX 109639, GeneTex). Following washes, the blots were incubated with horseradish peroxidase (HRP)-conjugated goat anti-mouse IgG (GTX 213111-01, GeneTex) and goat anti-rabbit IgG (GTX 213110-01, GeneTex) at a 1:5000 dilution for $2 \mathrm{~h}$. Detection of immunoreactive proteins was performed with an ECL system (Amersham Biosciences Corp., Piscataway, NJ, USA).

\subsection{Bacteriostatic Activity of Tg(ZP3:AN-hepc:ZP3:EGFP) Zebrafish Eggs by Disk Diffusion Assay}

One hundred fertilized eggs at $6 \mathrm{~h}$ postfertilization (hpf) from WT, Tg(ZP3:EGFP) and $\mathrm{Tg}$ (ZP3:AN-hepc:ZP3:EGFP) zebrafish, respectively, were collected in a 1.5-mL microcentrifuge tube containing $100 \mu \mathrm{L}$ of PBS and homogenized by a plastic homogenizer probe. After centrifuging at $17,500 \times \mathrm{g}$ for $15 \mathrm{~min}$ at $4{ }^{\circ} \mathrm{C}$ to collect the supernatant fraction, $100 \mu \mathrm{L}$ of the supernatant was loaded on an 8-mm filter disk (Tokyo Roshi Kaisha, Ltd., Japan) that was attached on the surface of a TBS agar plate containing a variety of indicator pathogens at a concentration of approximately $1.0 \times 10^{6} \mathrm{CFU} / \mathrm{mL}$. The TSB agar plate was incubated at $28^{\circ} \mathrm{C}$ for $24 \mathrm{~h}$, and then the diameter of the clear zone surrounding the filter disks was measured to evaluate the antimicrobial potency of the AN-hepc-expressing fish eggs.

\subsection{Fish Husbandry and Feeding Trial}

Two-month-old juvenile zebrafish (D. rerio) with an average body weight of $0.82 \pm 0.06 \mathrm{~cm}$ and body length of $3.86 \pm 0.36 \mathrm{~g}$ were randomly divided into three groups. Thirty fish per group were cultured in a 10-L acrylic tank at $28{ }^{\circ} \mathrm{C}$, and each group was tested in triplicate. The formulation and preparation of the basal diet, which comprised $39.2 \%$ crude protein and $8 \%$ crude lipid, were described in a previous report $[28,32]$. Fertilized zebrafish eggs at the one-cell stage used as a supplement were collected respectively from intercrosses of WT, F1 Tg(ZP3:EGFP) and F1 Tg(ZP3:AN-hepc:ZP3:EGFP). The fish were fed twice daily with the basal diet at $1 \%$ of body weight and supplemented with $150 \mathrm{WT}$ fertilized zebrafish eggs (WT group) or fed the basal diet and supplemented with $150 \mathrm{~F} 2 \mathrm{Tg}$ (ZP3:EGFP) fertilized eggs (Con group) or $150 \mathrm{~F} 2 \mathrm{Tg}$ (ZP3:AN-hepc:ZP3:EGFP) fertilized eggs (AN-hepc group) for 
two months. After two months feeding trial, innate immune parameter genes and pathogen challenge tests were evaluated.

\subsection{Detection of Innate Immune-Related Genes by Real-Time Polymerase Chain Reaction}

Six zebrafish from the WT, Con and AN-hepc groups were sampled, and total RNA from the whole bodies of the fish was extracted using TriPure isolation reagent (Roche, Mannheim, Germany). cDNA synthesis was carried out by an iScript cDNA Synthesis Kit (Bio-Rad, Hercules, CA, USA) according to the manufacturer's instructions. The expression levels of indicator genes involved in innate immune responses were determined by quantitative PCR (Applied Biosystems StepOnePlus, Foster City, CA, USA). The expression of elongation factor (EF)-1 $\alpha$ was used as an internal control. The specific primers used for detecting each gene are described in previous reports [28,33] and listed in Table 1. Quantitative PCR was conducted using SYBR Green PCR reagent, and the amplification program was carried out as follows: $60^{\circ} \mathrm{C}$ for $2 \mathrm{~min} ; 95^{\circ} \mathrm{C}$ for $10 \mathrm{~min}$; and 40 cycles of denaturing at $95^{\circ} \mathrm{C}$ for $15 \mathrm{~s}$, annealing and primer extension at $60^{\circ} \mathrm{C}$ for $1 \mathrm{~min}$. Equal quantities of total RNA from three fish were mixed and examined in triplicate for each condition. The relative expression level of each gene normalized to EF-1 $\alpha$ expression was expressed as the mean \pm standard error (S.E.).

Table 1. Primer sequences used in this study.

\begin{tabular}{|c|c|c|c|c|}
\hline Gene Name & Forward Primer Sequence & Reverse Primer Sequence & PCR Product Size (bp) & $\begin{array}{l}\text { Accession } \\
\text { Number }\end{array}$ \\
\hline Interleukin-1 $\beta$ (IL-1 $\beta)$ & TGGACTTCGCAGCACAAAATG & CACTTCACGCTCTTGGATGA & 147 & AY340959 \\
\hline Interleukin-6 (IL-6) & TCAACTTCTCCAGCGTGATG & ТСТTTCССТСТTТTССТССТG & 73 & JN698962 \\
\hline Interleukin-15 (IL-15) & ATGTCATTGGAACTCAGAGGTTT & CTGTTCTGGATGTCCTGCTTGA & 100 & BC162843 \\
\hline Tumor necrosis factor- $\alpha$ (TNF- $\alpha)$ & AAGGAGAGTTGCCTTTACCG & ATTGCCCTGGGTCTTATGC & 152 & BC165066 \\
\hline Nuclear factor kappa B (NF-kB) & AAGAGGACCAAAATAAGCACAG & TCCAAGGTACATCGCCATGA & 100 & AY163838 \\
\hline Complement component $\mathrm{C} 3 \mathrm{~b}$ & CGTCTCCGTACACCATCCATT & GGCGTCTCATCAGGATTTGTTAC & 100 & NM_131243 \\
\hline Lysozyme & CGTGGATGTCCTCGTGTGAAG & CCAATGGAGAATCCСТCAAA & 100 & NM_139180 \\
\hline Toll-like receptor-4a (TLR-4a) & TTTCAGATGCCACATCAGA & TCCACAAGAACAAGCCTTTG & 150 & EU551724 \\
\hline Elongation factor- $1 \alpha(E F-1 \alpha)$ & AACAGCTGATCGTTGGAGTCAA & TTGATGTATGCGCTGACTTCCT & 100 & AY422992 \\
\hline
\end{tabular}

\subsection{Evaluation of Disease Resistance by Challenge Test}

The cultivation, preparation and determination of the median lethal dose $\left(\mathrm{LD}_{50}\right)$ of the pathogens A. hydrophila and $S$. iniae for the challenge test were conducted according to a previously described protocol [28]. Briefly, A. hydrophila and S. iniae were cultured in TSB and incubated at $28^{\circ} \mathrm{C}$ for $24 \mathrm{~h}$. Bacterial cells were collected after centrifugation at $6100 \times g$ for $15 \mathrm{~min}$ at $4{ }^{\circ} \mathrm{C}$ and resuspended in distilled water to adjust the cell concentration. The 7-day LD50 was determined by intraperitoneal (i.p.) injection of serial doses of $A$. hydrophila and S. iniae separately $\left(10^{5}, 10^{6}, 10^{7}\right.$, and $10^{8} \mathrm{CFU} /$ fish) into 10 fish. At the end of the feeding experiment, the effect of AN-hepc-expressing zebrafish eggs on cumulative survival was evaluated by intraperitoneal (i.p.) injection of each fish with $20 \mu l$ of A. hydrophila and $S$. iniae solution (equal to the $\mathrm{LD}_{50}$ dose of $1.0 \times 10^{6} \mathrm{CFU}$ and $1 \times 10^{5} \mathrm{CFU}$ per fish, respectively). Fish fed the basal diet and WT zebrafish eggs and injected with saline or pathogens were used as the negative and positive controls, respectively. Injected fish (15 fish per tank) were cultured in a cylinder aquarium containing $10 \mathrm{~L}$ of fresh water at $28^{\circ} \mathrm{C}$. Each group was tested in triplicate. Challenged fish with infectious symptoms and dead fish were removed from the tanks. The cumulative survival in each group was recorded for 7 days post injection.

\subsection{Statistical Analysis}

Statistical differences in multiple-group comparisons of relative gene expression levels between groups were performed using one-way analysis of variance (ANOVA) and Tukey's multiple comparison tests. A probability value of less than $0.05(p<0.05)$ was considered a significant difference between the data presented in each group. Data analysis of cumulative survival in the challenge test was conducted by the Kaplan-Meier method using SAS software (SAS Institute, Cary, NC, USA). 


\section{Results}

\subsection{Establishment of Transgenic Zebrafish with Oocyte-Specific Expression of AN-hepc}

The expression plasmids pT2-ZP3-AN-hepc-ZP3-EGFP encoding recombinant A. nigrofasciata hepcidin (AN-hepc) and EGFP were triggered by the oocyte-specific zp3 promoter (Figure 1A). The chimeric plasmid utilized EGFP as a reporter to monitor the oocyte-specific expression of recombinant hepcidin, which included the first amino acid methionine at the N-terminus of the mature hepcidin and six histidine residues at the C-terminus for detecting hepcidin expression by a His-tag antibody (Figure 1B). In addition, the plasmid pT2-ZP3-EGFP encoding EGFP alone was used to establish a control transgenic line. Both plasmids (pT2-ZP3-AN-hepc-ZP3-EGFP and pT2-ZP3-EGFP) and Tol2 transposon mRNA were coinjected into fertilized zebrafish eggs at the one-cell stage to establish transgenic lines. Subsequently, the control transgenic lines Tg(ZP3:EGFP) and Tg(ZP3:AN-hepc:ZP3:EGFP) were successfully generated by Tol2 transposon-mediated integration of expression cassettes integrated into genomic DNA and characterized by EGFP specifically expressed in oocytes. The injected fish with genomic integration of the expression cassette were considered transgenic female founders (F0). EGFP expression was specifically located in the oocytes of F0 female Tg(ZP3:EGFP) and Tg(ZP3:AN-hepc:ZP3:EGFP) at one month post fertilization (Figure 2B,C). The F1 fertilized eggs from the intercrossed F0 transgenic fish exhibited maternal expression of EGFP protein and appeared intensely green under fluorescence (Figure 2E,F). These results potentially elucidated that AN-hepc is strongly expressed in F1 Tg(ZP3:AN-hepc:ZP3:EGFP) fertilized eggs and that transgenic fertilized eggs are a potential source of AN-hepc production.

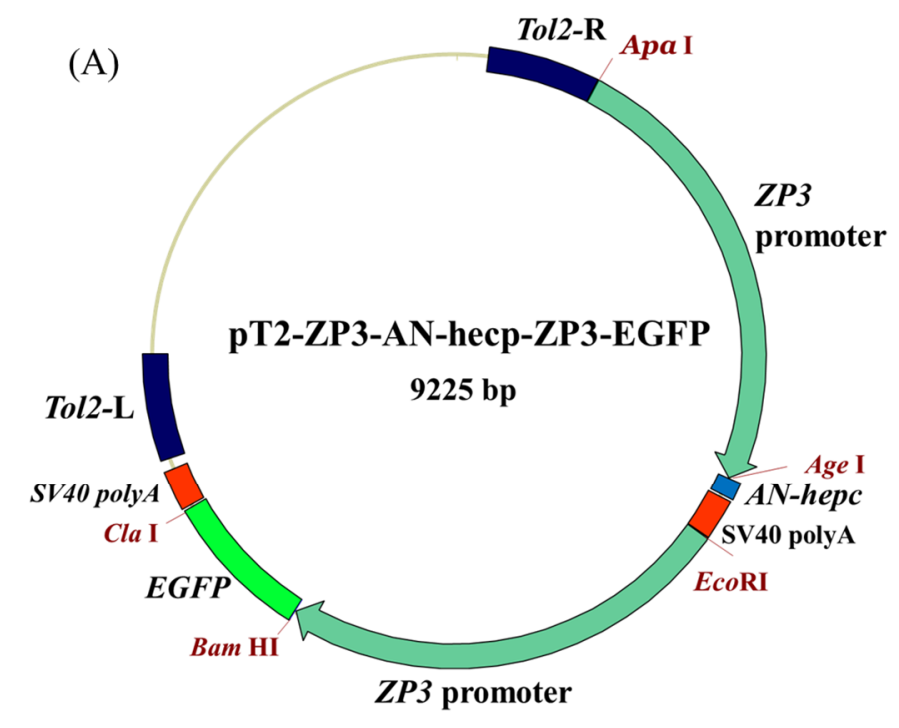

(B)

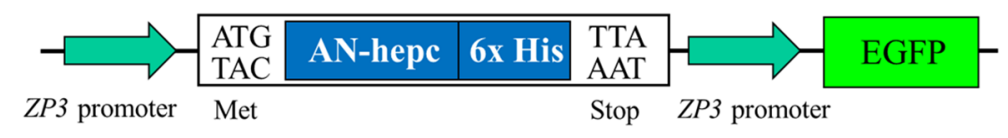

Figure 1. The expression plasmid pT2-ZP3-AN-hepc-ZP3-EGFP was used in this study. (A) The expression cassette of the pT2-ZP3-AN-hepc-ZP3-EGFP plasmid expressed AN-hepc and enhanced green fluorescent protein (EGFP) separately under the oocyte-specific zona pellucida ( $z$ 3) promoter. The Tol2 transposon elements were flanked with expression cassettes to enhance genomic integration. (B) EGFP was used as a reporter to monitor AN-hepc expression. Six histidine residues fused to the C-terminus of AN-hepc were easily detected by a His-tag antibody. 


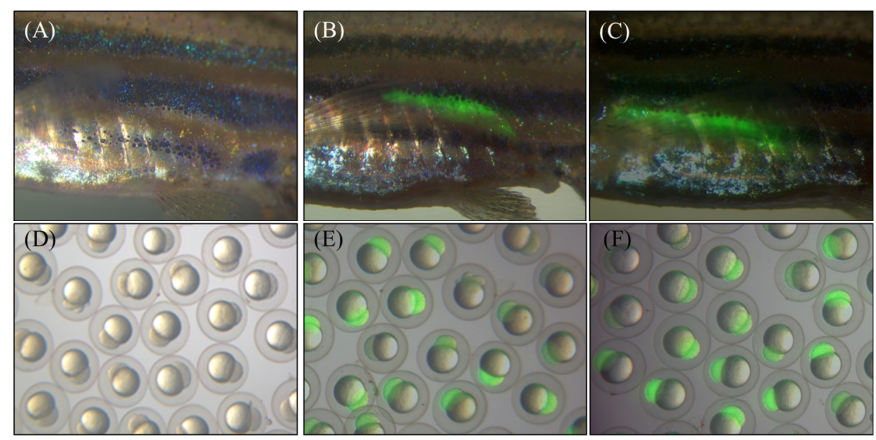

Figure 2. Transgenic zebrafish with oocyte-specific expression of AN-hepc and/or EGFP. (A) Wild-type zebrafish at 30 days post fertilization. (B and C) The expression of EGFP was specifically localized on the ovaries of F0 Tg(ZP3:EGFP) and Tg(ZP3:AN-hepc:ZP3:EGFP) at 30 days post fertilization. (D, E and F) F1 fertilized eggs harvested from intercrossed wild type, F1 Tg(ZP3:EGFP) and F1 $\operatorname{Tg}(\mathrm{ZP} 3: A N-h e p c: Z P 3: E G F P)$, respectively.

\subsection{Expression of AN-hepc in Transgenic Fish Eggs}

To confirm that the expression plasmids were integrated into F1 transgenic fish, the AN-hepc and EGFP genes were detected by PCR using genomic DNA as a template. The AN-hepc gene was only detected in F1 female $\operatorname{Tg}$ (ZP3:AN-hepc:ZP3:EGFP), and the EGFP gene was detected in Tg(ZP3:EGFP) and $\mathrm{Tg}(\mathrm{ZP} 3: \mathrm{AN}-\mathrm{hepc}: \mathrm{ZP} 3: \mathrm{EGFP})$, suggesting that genomic integration of the expression cassette had occurred in the F1 female transgenic line (Figure 3A). The mRNA expression of AN-hepc in F2 $\mathrm{Tg}$ (ZP3:AN-hepc:ZP3:EGFP) fertilized eggs was confirmed by RT-PCR. Naturally, the mRNA expression of EGFP was detected in EGFP-expressing F2 Tg(ZP3:EGFP) and $\mathrm{Tg}$ (ZP3:AN-hepc:ZP3:EGFP) fertilized eggs. The mRNA expression of the AN-hepc gene was detected in only $\mathrm{Tg}$ (ZP3:AN-hepc:ZP3:EGFP) fertilized eggs, suggesting that the AN-hepc gene was expressed in F2 Tg(ZP3:AN-hepc:ZP3:EGFP) fertilized eggs (Figure 3B). To further confirm the production of the AN-hepc peptide, 16.5\% Tricine-SDS-PAGE of soluble protein extracts from F2 Tg(ZP3:EGFP) and Tg(ZP3:AN-hepc:ZP3:EGFP) fertilized eggs was conducted and characterized by EGFP and the His-tag antibody. Similarly, EGFP was detected in F2 $\mathrm{Tg}$ (ZP3:EGFP) and $\mathrm{Tg}$ (ZP3:AN-hepc:ZP3:EGFP) fertilized eggs. A band with a size lower than $5 \mathrm{kDa}$ corresponding to the molecular weight of recombinant AN-hepc peptide was detected in the protein sample of $\mathrm{Tg}(\mathrm{ZP} 3: \mathrm{AN}-\mathrm{hepc}$ ZP3:EGFP) fertilized eggs by the His-tag antibody (Figure $3 C$ ). These results showed that EGFP could adequately reflect the extent of AN-hepc expression, and zebrafish eggs could be effectively used as bioreactors to produce the AN-hepc peptide

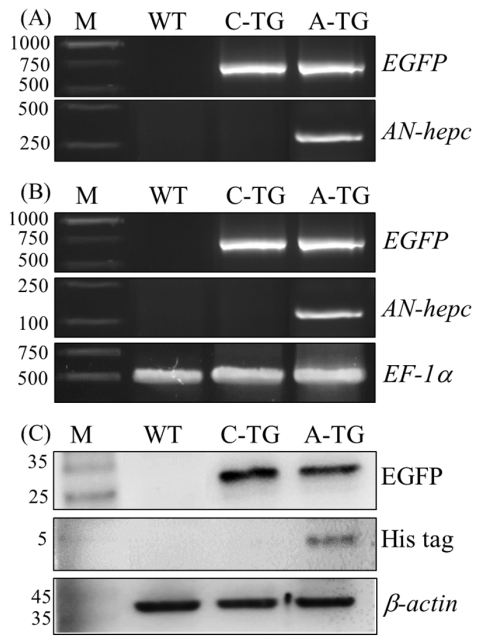

Figure 3. Detection of AN-hepc expression in fertilized eggs of Tg(ZP3:AN-hepc:ZP3:EGFP). 
(A) Genomic integration and germline transmission of the expression cassette in the F1 fertilized eggs of $\mathrm{Tg}$ (ZP3:AN-hepc:ZP3:EGFP) inherited from transgenic founders was confirmed by PCR. (B) AN-hepc mRNA expression was detected in fertilized eggs of F2 Tg(ZP3:AN-hepc:ZP3:EGFP) by RT-PCR. EF-1a was used as an internal control. (C) AN-hepc peptide produced in fertilized eggs of F2 $\mathrm{Tg}$ (ZP3:AN-hepc:ZP3:EGFP) was detected by western blotting using His-tag antibody. $\beta$-actin was used as an internal control. Lane M: molecular marker; lane WT: wild-type zebrafish; lane C-TG: Tg(ZP3:EGFP); lane A-TG: Tg (ZP3:AN-hepc:ZP3:EGFP).

\subsection{Antimicrobial Spectrum of the AN-hepc Peptide from Transgenic Fish Eggs}

Antimicrobial activity is an important indicator of antimicrobial peptide bioactivity. To confirm whether the fish egg-expressed AN-hepc exhibited biological activity, the antibacterial activity of cell-free extracts of transgenic zebrafish fertilized eggs was determined by a disk diffusion test. An obvious inhibitory zone was observed in the protein extract sample of F2 Tg(ZP3:AN-hepc:ZP3:EGFP) fertilized eggs, whereas samples from F2 $\mathrm{Tg}$ (ZP3:EGFP) fertilized eggs had no inhibitory zone (Supplementary Figure S1). The results demonstrated that AN-hepc expressed from transgenic zebrafish eggs is bioactive. The antimicrobial spectrum of AN-hepc from transgenic fish eggs was evaluated against a variety of pathogens. As shown in Table 2, the F2 Tg(ZP3:AN-hepc:ZP3:EGFP) fertilized eggs exhibited antimicrobial activity against the aquatic pathogens A. hydrophila, S. agalactiae, S. iniae, Vibrio alginolyticus, Vibrio parahaemolyticus, Vibrio vulnificus, and Debaryomyces hansenii. Notably, the AN-hepc peptide from F2 Tg(ZP3:AN-hepc:ZP3:EGFP) fertilized eggs exhibited antimicrobial potency against the antibiotic-resistant pathogens $V$. alginolyticus and $V$. parahaemolyticus, suggesting its potential as an alternative to antibiotics for treating antibiotic-resistant pathogenic infections (Table 2).

Table 2. Antimicrobial potency of the AN-hepc-expressing zebrafish eggs against various pathogens.

\begin{tabular}{lc}
\hline \multicolumn{1}{c}{ Pathogens } & Antimicrobial Activity ${ }^{\text {a }}$ \\
\hline Aeromonas hydrophila & +++ \\
Streptococcus agalactiae & ++ \\
Streptococcus iniae & +++ \\
Vibrio alginolyticus ${ }^{\text {b }}$ & ++ \\
Vibrio parahaemolyticus & ++ \\
Vibrio vulnificus & ++ \\
Debaryomyces hansenii & + \\
\hline
\end{tabular}

a Antimicrobial potency was determined by the disk diffusion test.,+++++ and + indicate clear zone diameters of $>1.2 \mathrm{~cm}$, between 1.0 and 1.2, and $<1.0 \mathrm{~cm}$, respectively. All assays were performed in triplicate. ${ }^{\mathrm{b}}$ indicates antibiotic-resistant pathogens.

\subsection{Supplementation with Zebrafish Eggs Containing AN-hepc Improves Innate Immunity}

The expression levels of immune-related genes, including interleukin (IL)-1 $\beta$, IL-6, IL-15, tumor necrosis factor (TNF)- $\alpha$, nuclear factor (NF)- $\mathrm{kB}$, complement component $3 \mathrm{~b}(\mathrm{C} 3 \mathrm{~b})$, lysozyme and toll-like receptor (TLR)- $4 \alpha$, were used as parameters to judge the health status of fish. The expression levels of IL-1 $\beta(p<0.001$ for AN-hepc vs. WT and AN-hepc vs. Con), IL-6 ( $p=0.002$ for AN-hepc vs. WT and AN-hepc vs. Con), IL-15 ( $p=0.002$ for AN-hepc vs. WT and AN-hepc vs. Con), TNF- $\alpha$ $(p<0.001$ for AN-hepc vs. WT and AN-hepc vs. Con), NF-kB ( $p=0.002$ for AN-hepc vs. WT and AN-hepc vs. Con), C3b ( $p=0.002$ for AN-hepc vs. WT and AN-hepc vs. Con), lysozyme ( $p<0.001$ for AN-hepc vs. WT and AN-hepc vs. Con) and TLR-4a ( $p<0.001$ for AN-hepc vs. WT and AN-hepc vs. Con) in fish from the AN-hepc group were significantly higher than those in fish from the WT and Con groups. The expression levels of the tested genes in fish were not significantly different between the WT and Con groups (Figure 4). The obvious induction in the expression of immune parameters in fish from the AN-hepc group suggested that supplementation with eggs expressing the AN-hepc antimicrobial peptide improved innate immunity in zebrafish. 

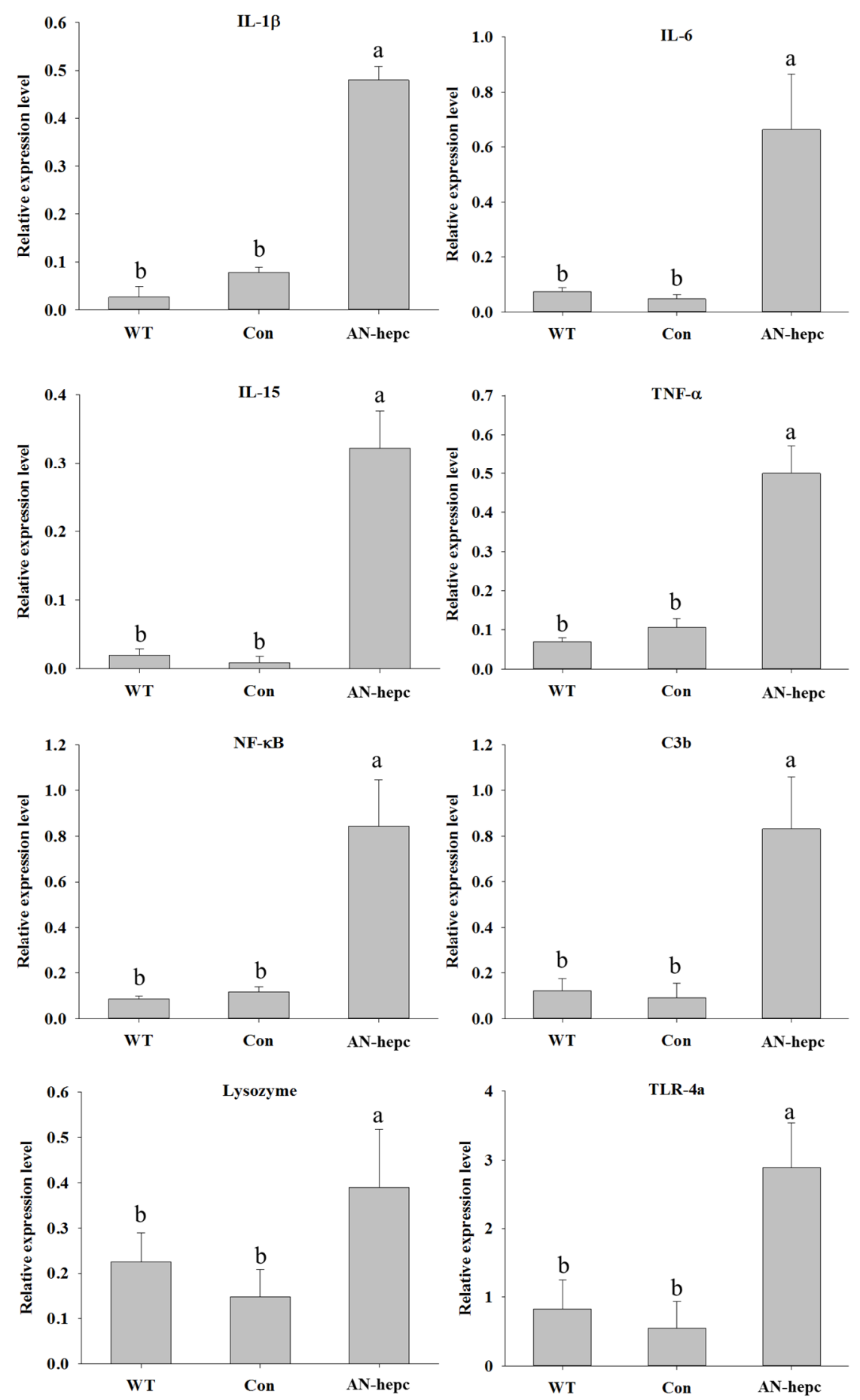

Figure 4. Relative expression levels of immune-related genes in zebrafish supplemented with wild-type zebrafish fertilized eggs (WT), Tg(ZP3:EGFP) fertilized eggs (Con) and Tg(ZP:AN-hepc:ZP3:EGFP) fertilized eggs (AN-hepc) for 2 months. (A) IL-1 $\beta$ (B) IL-6 (C) Il-15 (D) TNF- $\alpha$ (E) NF- $\mathrm{BB}$ (F) C3b (G) lysozyme $(\mathrm{H})$ TLR-4a. The data are presented as the mean \pm S.E. from six individual samples $(n=6)$. Values indicated by the bars with different letters indicate significant differences.

\subsection{Zebrafish Eggs Containing AN-hepc Enhance Disease Resistance}

Dietary supplementation with AN-hepc-expressing fish eggs improved innate immunity in zebrafish, prompting us to investigate the efficacy of AN-hepc on disease resistance against $A$. hydrophila and S. iniae. As shown in Figure 5, the survival rate at 7 days post infection was maintained at 98 
$\pm 3.84 \%$ and $100 \%$ for fish fed WT zebrafish eggs and injected with saline. The survival rate in zebrafish fed WT zebrafish eggs and injected with A. hydrophila or S. iniae dramatically declined during the first 4 days post infection, and then, a stable survival rate was maintained until 7 days post infection. The survival rates of fish in the WT and Con groups at 7 days post infection challenge with A. hydrophila or S. iniae were $24 \% \pm 3.84 \%$ and $27 \% \pm 6.67 \%$ or $13 \% \pm 6.67 \%$, and $18 \% \pm 3.84 \%$, respectively. The cumulative survival of fish exhibited no significant difference between the WT and Con groups challenged with $A$. hydrophila $(p=0.961)$ or $S$. iniae $(p=0.47)$. Notably, the survival rates of fish in the AN-hepc groups at 7 days post infection challenge with A. hydrophila or S. iniae were $48.9 \% \pm 3.84 \%$, and $48.8 \% \pm 6.67 \%$, respectively. The fish in the AN-hepc group exhibited an obviously increased survival rate at 7 days post infection with $A$. hydrophila or S. iniae compared to fish from the WT ( $p=0.029$ for A. hydrophila; $p=0.002$ for $S$. iniae) and Con groups ( $p=0.03$ for A. hydrophila; $p=0.005$ for $S$. iniae). The results suggested that fish supplemented with fish eggs containing AN-hepc showed enhanced disease resistance against $A$. hydrophila or $S$. iniae infection.
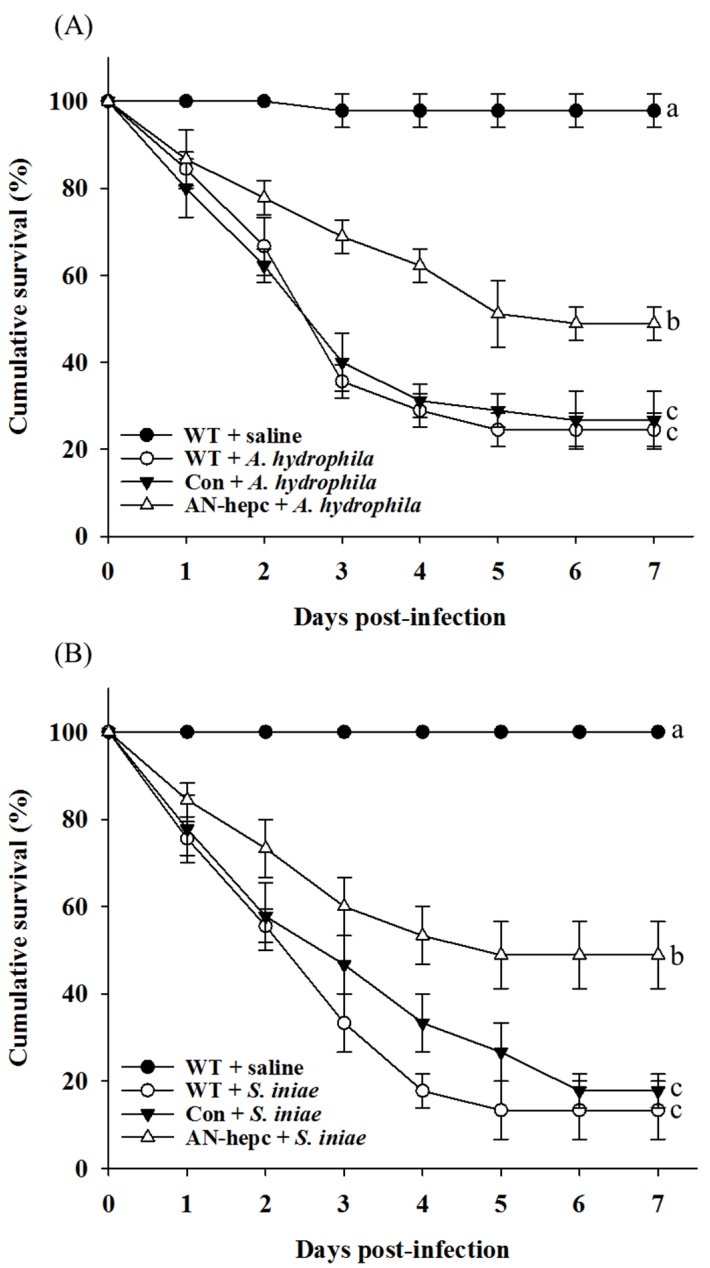

Figure 5. Cumulative survival rates of zebrafish challenged with (A) A. hydrophila and (B) S. iniae after supplemented with wild-type zebrafish fertilized eggs (WT), Tg(ZP3:EGFP) fertilized eggs (Con) and Tg(ZP:AN-hepc:ZP3:EGFP) fertilized eggs (AN-hepc) for 2 months. The cumulative survival curve with different letters indicates a significant difference according to Kaplan-Meier analysis.

\section{Discussion}

Prokaryotic expression systems such as E. coli bacteria are widely used for the production of heterologs; however, the production of the antimicrobial peptide hepcidin cannot be executed very successfully in E. coli. This may be attributed to the complex structure of the multiple disulfide bonds 
than prevent correct folding in the reductive environment of the cytoplasm and to the bactericidal activity, which is harmful to the E. coli host. Thus, hepcidin is usually produced as an inactive inclusion body in $E$. coli and requires complex renaturation procedures prior to the purification step [19]. Given this issue, an alternative strategy was required for hepcidin production. The present study established transgenic zebrafish in which AN-hepc was specifically expressed in eggs by the oocyte-specific $z p 3$ promoter. Unlike other cases in which recombinant proteins are ubiquitously expressed in transgenic fish $[24,25,34]$, the present study showed that AN-hepc can specifically accumulate during oogenesis; thus, fertilized eggs can be used as a source to produce AN-hepc without sacrificing fish. Expression of AN-hepc in transgenic fertilized eggs has been shown at the mRNA and protein levels by RT-PCR and Western blotting, demonstrating that AN-hepc is indeed produced in transgenic zebrafish eggs. In the present study, the biological activity of AN-hepc from transgenic fish eggs was confirmed by demonstrating its antimicrobial activity against diverse pathogens. Although AN-hepc was not purified from transgenic fish eggs, the cell-free supernatant from transgenic fish eggs exhibited antimicrobial activity, demonstrating that AN-hepc is produced as a soluble and bioactive protein in zebrafish eggs.

AMPs differ from conventional antibiotics and are often gene encoded and ribosomally synthesized peptides that respond to pathogens invading the innate immunity of the host. AMPs have been suggested as a potential alternative to classic antibiotics due to their immunomodulatory function and relatively low potential to elicit resistance [35]. In the present study, the AN-hepc-expressing fish eggs exhibited antimicrobial activity against aquatic pathogens and antibiotic-resistant aquatic pathogens, suggesting the potential of AN-hepc as an antimicrobial alternative to antibiotics in aquaculture. The present results showed that zebrafish fed AN-hepc-expressing eggs exhibited significantly increased cumulative survival after challenge with A. hydrophila and S. iniae, suggesting that supplementation with AN-hepc-expressing eggs can enhance resistance to pathogenic infections in fish. Although zebrafish is not a farmed fish in aquaculture, zebrafish have been widely have been used as model for several aquaculture-relevant infectious agents [36]. Investigations have reported that the probiotic immunostimulant exerts similar beneficial effects on zebrafish and Nile tilapia (O. niloticus), suggesting that the research performance in zebrafish could be reflected in farmed fish. Moreover, investigations have reported that grass carp (Ctenopharyngodon idellus) supplemented with recombinant Ctenopharyngodon idellus hepcidin (CiHep) could modulate immune responses and protect C. idellus against Flavobacterium columnare infection [37,38]. Grouper (Epinephelus lanceolatus) dietary supplemented with recombinant tilapia hepcidin 2-3 enhances immunity against Vibrio alginolyticus infection [39]. These results potentially implied the immunomodulatory function and protective effect of AN-hepc-expressing eggs on the zebrafish response to bacterial infection can be presented in farmed fish.

Biological compounds, such as $\beta$-Glucans, bioactive alginate, plant and animal extracts, vitamin $\mathrm{E}$ and probiotics, as immunostimulants have been widely studied in aquaculture for enhancing innate or non-specific cellular and humoral defense mechanism and preventing diseases in fish and shellfish. It is quite important to apply the immunostimulants at effective dosage. Treatment of immunostimulants overdose may cause adverse impacts in host [40]. In preliminary experiment, zebrafish supplement with 50, 100 and 150 of F2 Tg (ZP3:AN- hepc:ZP3:EGFP) fertilized eggs were evaluated in challenge test, respectively. Zebrafish reveled protect potency against $S$. iniae infection only in the presence of $150 \mathrm{~F} 2$ $\operatorname{Tg}$ (ZP3:AN-hepc:ZP3:EGFP) fertilized eggs, and exhibited healthy physiological state. Thus, the dose was choice to evaluate the immunomodulatory function on zebrafish. In this study, the expression of innate immune indicator genes in zebrafish after feeding with AN-hepc-expressing fish eggs was investigated to demonstrate the immunostimulatory function of AN-hepc. Cytokines secreted by specific immune cells act as intercellular messenger molecules in initiating the innate immune response to regulate the host defense against pathogenic infections. The proinflammatory cytokines IL-1 $\beta$, IL- 6 and TNF- $\alpha$ are produced predominantly by activated macrophages or monocytes during phagocytosis and assist the host in initiating an inflammatory response and activating immune cells to 
eliminate the invading pathogens. IL-15 secreted primarily from activated macrophages, monocytes and lymphocytes plays an important role in maintaining the development, differentiation, and survival of natural killer (NK) cells, which are bone marrow-derived lymphocytes that play crucial roles in the immune defense against pathogenic infections [41]. The transcription factor NF-kB acts as a central regulator of the proinflammatory signaling pathway to trigger the expression of cytokines, chemokines, and adhesion molecules and modulate innate immune functions [42]. Reports have shown that the NF-kB subunit regulates IL-6 transcription in flounder [43] and rainbow trout (Oncorhynchus mykiss) [44] and mediates TNF- $\alpha$-activated IL-6 expression in gilthead seabream (Sparus aurata) [45], suggesting the importance of NF- $\mathrm{kB}$ in teleost immunity. Moreover, TNF- $\alpha$ cloned from grass carp (Ctenopharyngodon idella) has been characterized as being involved in the NF- $\mathrm{kB}$ signaling pathway to regulate TNF- $\alpha$ and IL-1 $\beta$ expression in head kidney leukocytes, suggesting the importance of the link between TNF- $\alpha$ and NF- $\mathrm{KB}$ in modulating fish immunity [46]. The present study showed a significant increase in IL-1 $\beta$, IL-6, IL-15, TNF- $\alpha$ and NF- $\kappa B$ in zebrafish fed AN-hepc-expressing fish eggs, suggesting that AN-hepc-expressing fish eggs could modulate innate immunity. TLR-4 is a class of pattern recognition receptors (PRRs), which are highly conserved receptors that can recognize pathogen-associated molecular patterns (PAMPs). TLR-4 has been recognized as a sensor of gram-negative LPS; thus, it is presented as the first line of defense against infections. The complement system is made up of a series of distinct complement proteins that react with one another to augment the opsonization of pathogens by activating phagocytic cells and enhance the antibody-mediated killing of pathogens by attacking the cell membrane [47]. C3b, which is the main effector molecule of the complement system, acts as an opsonin that binds covalently to the surface of the pathogen and mediates the destruction of the pathogen by phagocytes [48]. Lysozyme produced by neutrophils and macrophages is an antimicrobial enzyme that plays a pivotal role in the prevention of bacterial infections by hydrolyzing the 1,4- $\beta$-linkages between $\mathrm{N}$-acetylmuramic acid and $\mathrm{N}$-acetyl-D-glucosamine in the peptidoglycan of the bacterial cell wall. Moreover, the lysozyme-mediated lysis of peptidoglycan can be recognized by PPRs, thereby activating phagocytosis and the complement system in the host [49]. The present results showing increased expression of the TLR-4a, C3b and lysozyme genes in zebrafish supplemented with AN-hepc-expressing eggs suggest that AN-hepc can improve lysozyme and complement activity in the innate immune system and enhance defensive recognition against pathogenic infection.

In conclusion, the present study demonstrated that AN-hepc was successfully expressed in transgenic fish eggs using an oocyte-specific promoter. The AN-hepc-expressing eggs exhibited antimicrobial bioactivity against diverse aquatic pathogens, suggesting the potential application of AN-hepc in aquaculture. Zebrafish supplemented with AN-hepc-expressing eggs exhibited not only increased expression of innate immune-related genes but also enhanced disease resistance to A. hydrophila and S. iniae infections. The present study is the first to use fish eggs to produce AN-hepc, and the results suggest that AN-hepc-expressing eggs could be used to produce AN-hepc and be developed as a supplement for immunomodulation in aquaculture.

Supplementary Materials: The following are available online at http:/www.mdpi.com/2071-1050/12/10/4057/ s1, Figure S1 Antibacterial activity of AN-hepc from transgenic fish eggs against (A) V. vulnificus and (B) methicllin-resistance S. aureus. (C) and (D) represented V. vulnificus and methicllin-resistance S. aureus resistant to ampicillin and kanamycin.

Author Contributions: Conceptualization, Conceptualization, S.-Y.H. and H.-T.S.; data curation, R.H.D. and Y.R.; formal analysis, R.H.D.; funding acquisition, S.-Y.H. and H.-T.S.; methodology, R.H.D. and T.-W.C.; resources, S.-Y.H. and C.-C.T.; supervision, S.-Y.H.; writing-Original draft, C.-C.T. and T.-W.C.; writing-Review and editing, S.-Y.H. All authors have read and agreed to the published version of the manuscript.

Funding: This study was supported by a grant from the Ministry of Science and Technology (MOST) 108-2313-B-020-005-MY3, Taiwan, and a grant from the Zuoying Branch of Kaohsiung Armed Forces General Hospital KAFGH-ZY-A-109031, Taiwan.

Conflicts of Interest: The authors declare no conflicts of interest. 


\section{References}

1. Mishra, A.; Nam, G.H.; Gim, J.A.; Lee, H.E.; Jo, A.; Kim, H.S. Current challenges of Streptococcus infection and effective molecular, cellular, and environmental control methods in aquaculture. Mol. Cells 2018, 41, 495-505. [PubMed]

2. Ina-Salwany, M.Y.; Al-Saari, N.; Mohamad, A.; Mursidi, F.A.; Mohd-Aris, A.; Amal, M.N.A.; Kasai, H.; Mino, S.; Sawabe, T.; Zamri-Saad, M. Vibriosis in fish: A review on disease development and prevention. J. Aquat. Anim. Health 2019, 31, 3-22. [CrossRef] [PubMed]

3. Katzenback, B.A. Antimicrobial peptides as mediators of innate immunity in teleosts. Biology 2015, 4, 607-639. [CrossRef] [PubMed]

4. Park, C.H.; Valore, E.V.; Waring, A.J.; Ganz, T. Hepcidin, a urinary antimicrobial peptide synthesized in the liver. J. Biol. Chem. 2001, 276, 7806-7810. [CrossRef]

5. Sangkhae, V.; Nemeth, E. Regulation of the iron homeostatic hormone hepcidin. Adv. Nutr. 2017, 8, $126-136$. [CrossRef]

6. Michels, K.; Nemeth, E.; Ganz, T.; Mehrad, B. Hepcidin and host defense against infectious diseases. PLoS Pathog. 2015, 11, e1004998. [CrossRef]

7. Aschemeyer, S.; Qiao, B.; Stefanova, D.; Valore, E.V.; Sek, A.C.; Ruwe, T.A.; Vieth, K.R.; Jung, G.; Casu, C.; Rivella, S.; et al. Structure-function analysis of ferroportin defines the binding site and an alternative mechanism of action of hepcidin. Blood 2018, 131, 899-910. [CrossRef]

8. Shike, H.; Lauth, X.; Westerman, M.E.; Ostland, V.E.; Carlberg, J.M.; Van Olst, J.C.; Shimizu, C.; Bulet, P.; Burns, J.C. Bass hepcidin is a novel antimicrobial peptide induced by bacterial challenge. Eur. J. Biochem. 2002, 269, 2232-2237. [CrossRef]

9. Banerjee, R.; Kanak, K.; Patel, B.; Samanta, M.; Das, S. Cloning and identification of antimicrobial peptide, hepcidin from freshwater carp, Catla catla on pathogen challenge and PAMPs stimulation. 3 Biotech 2019, 9, 341. [CrossRef]

10. Go, H.J.; Kim, C.H.; Park, J.B.; Kim, T.Y.; Lee, T.K.; Oh, H.Y.; Park, N.G. Biochemical and molecular identification of a novel hepcidin type 2-like antimicrobial peptide in the skin mucus of the pufferfish Takifugu pardalis. Fish Shellfish Immunol. 2019, 93, 683-693. [CrossRef]

11. Shirdel, I.; Kalbassi, M.R.; Hosseinkhani, S.; Paknejad, H.; Wink, M. Cloning, characterization and tissue-specific expression of the antimicrobial peptide hepcidin from caspian trout (Salmo caspius) and the antibacterial activity of the synthetic peptide. Fish Shellfish Immunol. 2019, 90, 288-296. [CrossRef] [PubMed]

12. Liu, Z.M.; Chen, J.; Lv, Y.P.; Hu, Z.H.; Dai, Q.M.; Fan, X.L. Molecular characterization of a hepcidin homologue in starry flounder (Platichthys stellatus) and its synergistic interaction with antibiotics. Fish Shellfish Immunol. 2018, 83, 45-51. [CrossRef] [PubMed]

13. Liu, Q.N.; Xin, Z.Z.; Zhang, D.Z.; Jiang, S.H.; Chai, X.Y.; Wang, Z.F.; Li, C.F.; Zhou, C.L.; Tang, B.P. cDNA cloning and expression analysis of a hepcidin gene from yellow catfish Pelteobagrus fulvidraco (Siluriformes: Bagridae). Fish Shellfish Immunol. 2017, 60, 247-254. [CrossRef] [PubMed]

14. Xu, G.; Huang, T.; Gu, W.; Zhang, Y.; Yao, Z.; Zhao, C.; Wang, B. Characterization, expression, and functional analysis of the hepcidin gene from Brachymystax lenok. Dev. Comp. Immunol. 2018, 89, 131-140. [CrossRef] [PubMed]

15. Jiang, X.F.; Liu, Z.F.; Lin, A.F.; Xiang, L.X.; Shao, J.Z. Coordination of bactericidal and iron regulatory functions of hepcidin in innate antimicrobial immunity in a zebrafish model. Sci. Rep. 2017, 7, 4265. [CrossRef] [PubMed]

16. Shen, Y.; Zhao, Z.; Zhao, J.; Chen, X.; Cao, M.; Wu, M. Expression and functional analysis of hepcidin from mandarin fish (Siniperca chuatsi). Int. J. Mol. Sci. 2019, 20, 5602. [CrossRef] [PubMed]

17. Chen, S.L.; Xu, M.Y.; Ji, X.S.; Yu, G.C.; Liu, Y. Cloning, characterization, and expression analysis of hepcidin gene from red sea bream (Chrysophrys major). Antimicrob. Agents Chemother. 2005, 49, 1608-1612. [CrossRef]

18. Chi, J.R.; Liao, L.S.; Wang, R.G.; Jhu, C.S.; Wu, J.L.; Hu, S.Y. Molecular cloning and functional characterization of the hepcidin gene from the convict cichlid (Amatitlania nigrofasciata) and its expression pattern in response to lipopolysaccharide challenge. Fish Physiol. Biochem. 2015, 41, 449-461. [CrossRef]

19. Zhang, H.; Yuan, Q.; Zhu, Y.; Ma, R. Expression and preparation of recombinant hepcidin in Escherichia coli. Protein Expr. Purif. 2005, 41, 409-416. [CrossRef] 
20. Da Costa, A.; Pereira, A.M.; Gomes, A.C.; Rodriguez-Cabello, J.C.; Casal, M.; Machado, R. Production of bioactive hepcidin by recombinant DNA tagging with an elastin-like recombinamer. New Biotechnol. 2018, 46, 45-53. [CrossRef]

21. Sadr, V.; Saffar, B.; Emamzadeh, R. Functional expression and purification of recombinant hepcidin 25 production in Escherichia coli using SUMO fusion technology. Gene 2017, 610, 112-117. [CrossRef] [PubMed]

22. Pan, C.Y.; Huang, T.C.; Wang, Y.D.; Yeh, Y.C.; Hui, C.F.; Chen, J.Y. Oral administration of recombinant epinecidin-1 protected grouper (Epinephelus coioides) and zebrafish (Danio rerio) from Vibrio vulnificus infection and enhanced immune-related gene expressions. Fish Shellfish Immunol. 2012, 32, 947-957. [CrossRef] [PubMed]

23. Tao, Y.; Zhao, D.M.; Wen, Y. Expression, purification and antibacterial activity of the channel catfish hepcidin mature peptide. Protein Expr. Purif. 2014, 94, 73-78. [CrossRef] [PubMed]

24. Hwang, G.; Muller, F.; Rahman, M.A.; Williams, D.W.; Murdock, P.J.; Pasi, K.J.; Goldspink, G.; Farahmand, H.; Maclean, N. Fish as bioreactors: Transgene expression of human coagulation factor VII in fish embryos. Mar. Biotechnol. 2004, 6, 485-492. [CrossRef] [PubMed]

25. Lin, C.Y.; Yang, P.H.; Kao, C.L.; Huang, H.I.; Tsai, H.J. Transgenic zebrafish eggs containing bactericidal peptide is a novel food supplement enhancing resistance to pathogenic infection of fish. Fish Shellfish Immunol. 2010, 28, 419-427. [CrossRef]

26. Hu, S.Y.; Liao, C.H.; Lin, Y.P.; Li, Y.H.; Gong, H.Y.; Lin, G.H.; Kawakami, K.; Yang, T.H.; Wu, J.L. Zebrafish eggs used as bioreactors for the production of bioactive tilapia insulin-like growth factors. Transgen. Res. 2011, 20, 73-83. [CrossRef]

27. Tseng, C.C.; Murni, L.; Han, T.W.; Arfiati, D.; Shih, H.T.; Hu, S.Y. Molecular characterization and heterologous production of the bacteriocin peocin, a DNA starvation/stationary phase protection protein, from Paenibacillus ehimensis NPUST1. Molecules 2019, 24, 2516. [CrossRef]

28. Yi, C.C.; Liu, C.H.; Chuang, K.P.; Chang, Y.T.; Hu, S.Y. A potential probiotic Chromobacterium aquaticum with bacteriocin-like activity enhances the expression of indicator genes associated with nutrient metabolism, growth performance and innate immunity against pathogen infections in zebrafish (Danio rerio). Fish Shellfish Immunol. 2019, 93, 124-134. [CrossRef]

29. Chen, S.W.; Liu, C.H.; Hu, S.Y. Dietary administration of probiotic Paenibacillus ehimensis NPUST1 with bacteriocin-like activity improves growth performance and immunity against Aeromonas hydrophila and Streptococcus iniae in Nile tilapia (Oreochromis niloticus). Fish Shellfish Immunol. 2019, 84, 695-703. [CrossRef]

30. Hu, S.Y.; Lin, P.Y.; Liao, C.H.; Gong, H.Y.; Lin, G.H.; Kawakami, K.; Wu, J.L. Nitroreductase-mediated gonadal dysgenesis for infertility control of genetically modified zebrafish. Mar. Biotechnol. 2010, 12, 569-578. [CrossRef]

31. Kawakami, K. Transgenesis and gene trap methods in zebrafish by using the Tol2 transposable element. Methods Cell Biol. 2004, 77, 201-222. [PubMed]

32. Lin, Y.S.; Saputra, F.; Chen, Y.C.; Hu, S.Y. Dietary administration of Bacillus amyloliquefaciens R8 reduces hepatic oxidative stress and enhances nutrient metabolism and immunity against Aeromonas hydrophila and Streptococcus agalactiae in zebrafish (Danio rerio). Fish Shellish Immunol. 2019, 86, 410-419. [CrossRef] [PubMed]

33. Faikoh, E.N.; Hong, Y.H.; Hu, S.Y. Liposome-encapsulated cinnamaldehyde enhances zebrafish (Danio rerio) immunity and survival when challenged with Vibrio vulnificus and Streptococcus agalactiae. Fish Shellfish Immunol. 2014, 38, 15-24. [CrossRef] [PubMed]

34. Morita, T.; Yoshizaki, G.; Kobayashi, M.; Watabe, S.; Takeuchi, T. Fish eggs as bioreactors: The production of bioactive luteinizing hormone in transgenic trout embryos. Transgen. Res. 2004, 13, 551-557. [CrossRef] [PubMed]

35. Roncevic, T.; Puizina, J.; Tossi, A. Antimicrobial peptides as anti-infective agents in pre-post-antibiotic era? Int. J. Mol. Sci. 2019, 20, 5713. [CrossRef]

36. Lee-Estevez, M.; Figueroa, E.; Cosson, J.; Short, S.E.; Valdebenito, I.; Ulloa-Rodríguez, P.; Farías, J.G. Zebrafish as a useful model for immunological research with potential applications in aquaculture. Rev. Aquacu. 2016, 10, 213-223. [CrossRef]

37. Wei, X.; Sarath Babu, V.; Lin, L.; Hu, Y.; Zhang, Y.; Liu, X.; Su, J.; Li, J.; Zhao, L.; Yuan, G. Hepcidin protects grass carp (Ctenopharyngodon idellus) against Flavobacterium columnare infection via regulating iron distribution and immune gene expression. Fish Shellfish Immunol. 2018, 75, 274-283. [CrossRef] 
38. Chen, T.; Zhou, J.; Qu, Z.; Zou, Q.; Liu, X.; Su, J.; Fu, X.; Yuan, G. Administration of dietary recombinant hepcidin on grass carp (Ctenopharyngodon idella) against Flavobacterium columnare infection under cage aquaculture conditions. Fish Shellfish Immunol. 2020, 99, 27-34. [CrossRef]

39. Ting, C.H.; Pan, C.Y.; Chen, Y.C.; Lin, Y.C.; Chen, T.Y.; Rajanbabu, V.; Chen, J.Y. Impact of Tilapia hepcidin 2-3 dietary supplementation on the gut microbiota profile and immunomodulation in the grouper (Epinephelus lanceolatus). Sci. Rep. 2019, 9, 19047. [CrossRef]

40. Farooqi, F.S.; Qureshi, W.U.H. Immunostimulants for aquaculture health management. J. Pharm. Phytochem. 2018, 7, 1441-1447.

41. Perera, P.Y.; Lichy, J.H.; Waldmann, T.A.; Perera, L.P. The role of interleukin-15 in inflammation and immune responses to infection: Implications for its therapeutic use. Microbes Infect. 2012, 14, 247-261. [CrossRef] [PubMed]

42. Mussbacher, M.; Salzmann, M.; Brostjan, C.; Hoesel, B.; Schoergenhofer, C.; Datler, H.; Hohensinner, P.; Basilio, J.; Petzelbauer, P.; Assinger, A.; et al. Cell type-specific roles of NF-kappaB linking inflammation and thrombosis. Front. Immunol. 2019, 10, 85. [CrossRef] [PubMed]

43. Kong, H.J.; Nam, B.H.; Kim, Y.O.; Kim, W.J.; Cho, H.K.; Lee, C.H.; Lee, S.J.; Kim, K.K. Characterization of the flounder IL-6 promoter and its regulation by the p65 NF-kappaB subunit. Fish Shellfish Immunol. 2010, 28, 961-964. [CrossRef] [PubMed]

44. Zante, M.D.; Borchel, A.; Brunner, R.M.; Goldammer, T.; Rebl, A. Cloning and characterization of the proximal promoter region of rainbow trout (Oncorhynchus mykiss) interleukin-6 gene. Fish Shellfish Immunol. 2015, 43, $249-256$. [CrossRef] [PubMed]

45. Castellana, B.; Marin-Juez, R.; Planas, J.V. Transcriptional regulation of the gilthead seabream (Sparus aurata) interleukin-6 gene promoter. Fish Shellfish Immunol. 2013, 35, 71-78. [CrossRef] [PubMed]

46. Zhang, A.; Chen, D.; Wei, H.; Du, L.; Zhao, T.; Wang, X.; Zhou, H. Functional characterization of TNF-alpha in grass carp head kidney leukocytes: Induction and involvement in the regulation of NF-kappaB signaling. Fish Shellfish Immunol. 2012, 33, 1123-1132. [CrossRef]

47. Rus, H.; Cudrici, C.; Niculescu, F. The role of the complement system in innate immunity. Immunol. Res. 2005, 33, 103-112. [CrossRef]

48. Boshra, H.; Li, J.; Sunyer, J.O. Recent advances on the complement system of teleost fish. Fish Shellfish Immunol. 2006, 20, 239-262. [CrossRef]

49. Ragland, S.A.; Criss, A.K. From bacterial killing to immune modulation: Recent insights into the functions of lysozyme. PLoS Pathog. 2017, 13, e1006512. [CrossRef] 